H I G H L I G H T S

TUMORIGENESIS

\title{
High-level Hedgehog hazard
}

The Hedgehog (HH) signalling pathway is active in various embryonic tissues but reactivation of this pathway in adult tissue is associated with skin, cerebral and skeletal-muscle tumours. HH has a particularly dominant role in the development and homeostasis of gut tissue and now two papers published in Nature from Sarah P. Thayer et al. and David M. Berman et al. - show that $\mathrm{HH}$ pathway activation might also cause gastrointestinal tumours.

Berman et al. found that $81 \%$ of gut-derived tumour cell lines - from oesophageal, stomach, biliary and pancreatic tumours - expressed sonic hedgehog $(\mathrm{SHH})$ and its receptor PTCH, which was also highly expressed in resected pancreatic and stomach tumours. Treatment of PTCH-expressing cell lines with the $\mathrm{HH}$-pathway antagonist cyclopamine inhibited proliferation. Furthermore, cyclopamine-treatment of PTCHexpressing tumour cells transfected with a GLI-reporter construct another downstream target of $\mathrm{HH}-$ abolished luciferase activity, indicating that the $\mathrm{HH}$ pathway is involved in tumour growth. When they blocked endogenous $\mathrm{HH}$ in tumour cell cultures, by adding an $\mathrm{HH}$ monoclonal antibody at concentrations just sufficient to prevent growth, addition of increasing concentrations of exogenous SHH ligand enhanced cell proliferation. So, tumour growth is driven by endogenous expression of $\mathrm{SHH}$, which activates the $\mathrm{HH}$ pathway.

Thayer et al. examined pancreata from transgenic mice misexpressing Shh and found abnormal epithelial changes resembling the precursor lesions of pancreatic cancer. The lesions contained mutations in Kras and overexpressed Erbb2 (also known as Her2/neu) — alterations found in early-stage human pancreatic cancers. PTCH expression was not detected in normal tissue but was detected throughout the abnormal epithelium of murine and human neoplastic pancreata, so they too investigated whether the $\mathrm{HH}$ pathway is involved in pancreatic tumorigenesis. Similar to the studies by Berman et al., they treated pancreatic tumour cell lines with cyclopamine, which inhibited proliferation in $50 \%$ of the cell lines expressing the $\mathrm{HH}$ target $\mathrm{SMO}$. In the other $50 \%$, mutations in the $\mathrm{HH}$ pathway downstream of SMO might contribute to aberrant proliferation or, alternatively, $\mathrm{HH}$-independent pathways might be mutated. GLI-reporter assays confirmed that inactivation of the $\mathrm{HH}$ pathway inhibited pancreatic cancer cell proliferation.

Both groups then investigated the in vivo effects of cyclopamine using tumorigenic mouse models. Berman et al. established human cholangiocarcinoma tumour cell xenografts in athymic nude mice and cyclopamine caused complete regression of $\sim 180$ $\mathrm{mm}^{3}$ tumours. Thayer et al. tested the ability of cyclopamine to inhibit proliferation of cyclopamine-responsive pancreatic tumour cell lines L3.6sl and Panc 05.04 — injected into CD-1 nude mice. Cyclopamine, administered when tumours were palpable, caused a $50-60 \%$ reduction in the tumour-cell mass of both cell lines, and when given at the time of cell implant the L3.6sl tumour mass was reduced by $84 \%$. These separate observations confirm that blocking the $\mathrm{HH}$ pathway in vivo can cause tumour regression.

Collectively, both studies show that the $\mathrm{HH}$ signalling pathway has an important role in certain digestive tract tumours and that $\mathrm{HH}$ ligand expression identifies a group of common lethal malignancies. Whether this pathway is activated in other forms of cancer remains to be established.

Emma Croager

\section{(1) References and links}

ORIGINAL RESEARCH PAPERS Thayer, S. et al. Hedgehog is an early and late mediator of pancreatic cancer tumorigenesis. Nature $14 \mathrm{Sept}$ 2003 (doi:10.1038/nature02009) | Berman, D. M. et al. Widespread requirement for Hedgehog ligand stimulation in growth of digestive tract tumours. Nature 14 Sept 2003 (doi:10.1038/nature01972) WEB SITES

Matthias Hebrok's lab:

http://diabetes.ucsf.edu/hebrok/ Philip A. Beachy's lab:

http://www.hhmi.org/research/investigators/beac hy.html

\section{IN BRIEF}

METASTASIS

A causal role for endothelin-1 in the pathogenesis of osteoblastic bone metastases.

Yin, J. J. et al. Proc. Natl Acad. Sci. USA 26 Aug 2003

(doi:10.1073/pnas.1830978100)

Yin et al. have developed a reproducible model of osteoblastic metastases by inoculating nude mice with ZR-75-1 human breast cancer cells, which secrete endothelin-1 (ET-1). Treatment of these mice with an ET-receptor antagonist markedly decreased metastases and tumour burden in bone. As ZR-75-1 cells do not express ET-1 receptor, the authors propose that the effects of ET-1 in this model are mediated through osteoblasts.

\section{TUMORIGENESIS}

TSC2 regulates VEGF through mTOR-dependent and -independent pathways.

Brugarolas, J. B. et al. Cancer Cell 4, 147-158 (2003)

Tuberous sclerosis complex (TSC) - a hereditary cancer syndrome caused by mutations in TSC1 or TSC2 - is characterized by highly vascular tumours. The TSC1-TSC2 complex negatively regulates mammalian target of rapamycin (mTOR) and HIF. Brugarolas et al. found increased levels of Hif and its target Vegf in $\mathrm{TSC}^{-1-}$ mouse embryonic fibroblasts (MEFs). The mTOR antagonist rapamycin normalises Hif levels in Tsc2 ${ }^{-/-}$MEFs, but only partially inhibits Vegf expression, indicating that TSC2 regulates VEGF through mTOR-dependent and -independent pathways.

\section{EPIGENETICS}

Genetic unmasking of epigenetically silenced tumor suppressor genes in colon cancer cells deficient in DNA methyltransferases.

Paz, M. F. et al. Hum. Mol. Genet. 12, 2209-2219 (2003)

Tumour-suppressor genes are often silenced in tumours by hypermethylation of the $\mathrm{CpG}$ islands within the genes. To find out which genes were silenced in colon cancer, the authors knocked out two key DNA methyltransferase genes - DNMT1 and DNMT3b - in a colon cancer cell line. Loss of hypermethylated $\mathrm{CpG}$ islands induced the reactivation of many known tumorigenesis-associated genes, such as the cadherin family member FAT and the homeobox genes LMX1 and DUX4. They also found other genes, including a calcium-channel subunit that had not previously been shown to have a role in tumorigenesis.

\section{ANTISENSE THERAPY}

Pharmacologic inactivation of kinase suppressor of ras-1 abrogates Ras-mediated pancreatic cancer.

Xing, H. R. et al. Nature Med. 7 Sept 2003 (doi:10.1038/nm927)

The growth of pancreatic cancer, for which there is no effective therapy, is RAS dependent. Xing et al. used an antisense oligonucleotide to the kinase suppressor of RAS-1 (KSR1) to prevent growth of established human pancreatic xenografts in nude mice, without any toxic effects. 